\title{
A Study on After effects of Holi: Color Injury, Ocular Trauma, and Assault at a Tertiary Care Ophthalmic Center in North India
}

\author{
${ }^{1}$ Arushee Bhatnagar, ${ }^{2}$ Shakti K Gupta, ${ }^{3}$ Vikas Hanumanthappa, ${ }^{4}$ Nitin Garg
}

\begin{abstract}
Introduction: India is known for its diverse culture and religions which are indeed famous for their variety of festivals. The presented study deals with one such festival called Holi and its hazardous ocular effects at Tertiary Care Ophthalmic Center In North India.
\end{abstract}

Aim: To study the demographic and clinical profile of patients visiting ophthalmologic emergency tertiary care ophthalmic center during Holi in New Delhi.

\section{Objectives:}

- To study the pattern of normal patient flow in the emergency department of RPC.

- To study the demographic and clinical profile of patients visiting Emergency Department during Holi.

- To study the trend of Holi cases over the years.

Materials and methods: The data were collected by conducting direct interviews with patients and from medical records of the patients visiting the Emergency Department of RPC from March 1 to 5, 2018 (i.e., 5 days). These 5 days were chosen so that it could cover the pre-Holi day, Holi day and post-Holi day. Data of total 210 patients who visited the Emergency department of RPC, AIIMS, New Delhi, were taken. Statistical data were analyzed using Microsoft Excel.

Results and discussion: An increase in awareness among people shows a substantial decline in number of color injury, complications, traumatic eye injuries, and assault cases from the year 2016. Men (20) were more affected than women (9), with most common being among the youth (21 years \pm 10 ). No change in routine cases was recorded in normal patient flow to the casualty, but a sudden spike in cases of color injury (29) was observed during the study period. Sooner the patient reported, better was the prognosis.

Keywords: Chemical injury, Emergency, Holi, Ocular injury, Ocular trauma, Substance abuse.

\footnotetext{
${ }^{1}$ Intern, ${ }^{2}$ Medical Superintendent, ${ }^{3}$ Senior Resident, ${ }^{4}$ Junior Resident

${ }^{1}$ Bharati Vidyapeeth (Deemed to be University) Medical College Pune, Maharashtra, India

${ }^{2-4}$ All India Institute of Medical Sciences, Dr. R P Centre for Ophthalmic Sciences, New Delhi, India

Corresponding Author: Arushee Bhatnagar, Intern, Bharati Vidyapeeth (Deemed to be University) Medical College, Pune Maharashtra, India, e-mail: arusheemedico999@gmail.com
}

How to cite this article: Bhatnagar A, Gupta SK, Hanumanthappa V, Garg N. A Study on After effects of Holi: Color Injury, Ocular Trauma, and Assault at a Tertiary Care Ophthalmic Center in North India. Int J Res Foundation Hosp Healthc Adm 2018;6(1):31-36.

\section{Source of support: Nil}

\section{Conflict of interest: None}

\section{INTRODUCTION}

India is one of the most diverse countries in the world harboring people belonging to different religions and caste. Every religion has it has its own cultural and traditional festivals which are celebrated in unique ways. The major religious festivals are Holi, RakshaBandhan, Diwali, Janamastami, Durga-Puja, Dussehra, Eid, Christmas, etc. ${ }^{1}$

Holi is a traditional Hindu festival originated in India. People celebrate it by playing with Gulal, a special colored powder, at each other. This Holi color/Gulal powder is manufactured by different companies. The quality and the amount of information given on the outer packaging about the ingredients of the particular Holi color are mostly very poor.

Ophthalmology is a branch of medicine which deals with diagnosis, treatment, and prophylaxis of diseases in the eye and visual pathway. ${ }^{2}$

Every year during the festive period, there is a sudden spike in cases of chemical injury, assault, and trauma cases in the eye. Hence, this study was chosen in order to look for the trend and its variation for the year 2018.

The severity of injury related to colors depends on composition, amount, concentration, time since exposure, and duration of reporting to a hospital from the time of incident. ${ }^{3}$ The immediate symptoms can be blurred vision/blindness, inflammatory condition, allergic reaction, epiphora, and dermatitis. Balloon injury is the most common type of ocular trauma. They are being hit randomly by strangers from heights as a part of prank and fun which can cause blunt eye injuries. There can be hyphema, lens subluxation, macular edema, or retinal detachment, leading to loss of vision, rupture of blood vessels, diminished vision, fracture, and bruising. ${ }^{4}$ Another common example of ocular trauma is conflict 
related. Cases of physical assaults and road accidents are recorded mainly under the influence of substance abuse like hallucinogens more commonly being "bhaang," "charas," marijuana, cocaine, and alcohol. Bhang is exclusively sold during Holi which is a hallucinogen under whose influence the person forgets about the time, events that have occurred during that interval. ${ }^{5}$ Blindness resulting under such circumstances is classified as preventable blindness. It is defined as a blindness which could be either treated or prevented before the occurrence of permanent damage. ${ }^{6}$

\section{REVIEW OF LITERATURE}

A study was conducted by Bossmann et $\mathrm{al}^{7}$ on composition of Holi colors, who interpreted that an aerodynamic diameter smaller than $10 \mu \mathrm{m}$, so-called PM10 particles, can induce proinflammatory response and an oxidative leukocyte burst, after analyzing four different Holi colors regarding their particle size.

A review study conducted by Susman ${ }^{8}$ on water balloon slingshots concluded that the balloons can be thrown at a speed up to 94 miles per hour which can cause rupture of blood vessels in eyes, diminished vision, hemorrhage, or fracture of the orbital bone surrounding the eye.

Another study by Velpandian et $\mathrm{al}^{9}$ analyzed about ocular hazards caused by malachite green in Holi colors and evaluated the corneal penetration of malachite green using goat cornea in perfusion chamber and concluded that malachite green was extensively used in our festival of Holi and has caused severe ocular irritation with epithelial defect upon exposure.

A study by Ghose et $\mathrm{al}^{3}$ carried out a prospective study on ocular chemical injury, which suggested that out of 50 cases of acute chemical burns, $86 \%$ of them were men with a mean age of 25 years who were most affected.

\section{MATERIALS AND METHODS}

A descriptive cross-sectional observational study was conducted at the RPC in New Delhi in the Casualty Department of Ophthalmology for a period of 5 days to assess the patient flow during the festive period of Holi.

\section{Inclusion Criterion}

Patients reporting to the Emergency Department during the duration of the festival, i.e., from March 1 to 5, 2018.

\section{Exclusion Criterion}

Patients who reported before March 1 and after March 5, 2018.

\section{Study Period}

It was divided into three phases:

1. Phase I

2. Phase II

3. Phase III

Phase I (March 1, 2018) dealt with all the pre-Holi cases (43), phase II (March 2, 2018) included cases which happened on the day of Holi (45), and phase III (March $3-5,2018)$ dealt with the post-Holi cases (122), i.e., after the day of Holi for 3 days.

Direct open interviews were conducted with the patients and their relatives to record the significant demographic data. Patients' records were also reviewed for additional information. The data collected were analyzed using Microsoft Excel.

\section{OBSERVATIONS}

Ophthalmic casualty hosts a variety of patients everyday viz., trauma, glaucoma, foreign body penetration, cataract, etc.

The patients' record is maintained by the nurses in three shifts: from 12 am to $1 \mathrm{pm}$, from $1 \mathrm{pm}$ to $7 \mathrm{pm}$, and from $7 \mathrm{pm}$ to $12 \mathrm{am}$. In normal routine days, the patients in need of immediate care directly report to the doctor, and in case of delay, they are asked to report to the nurse, where the history is recorded and any preliminary treatment is provided. Minor operation theater is used for suturing or for administration of anesthesia next to the casualty, whereas minor procedures like intravitreal injections, measurement of intraocular pressure (IOP), and saline wash, and are done on bedside within the casualty.

During Holi, the department is pre-prepared for patients (Fig. 1), with full-time availability of emergency drugs, additional staff on station and special eye drops

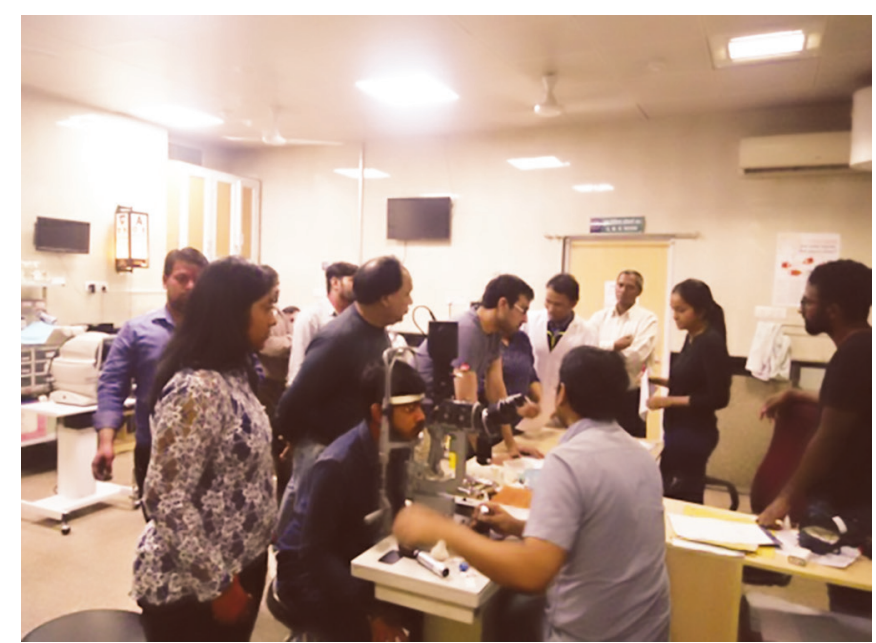

Fig. 1: Casualty scenario on Holi 


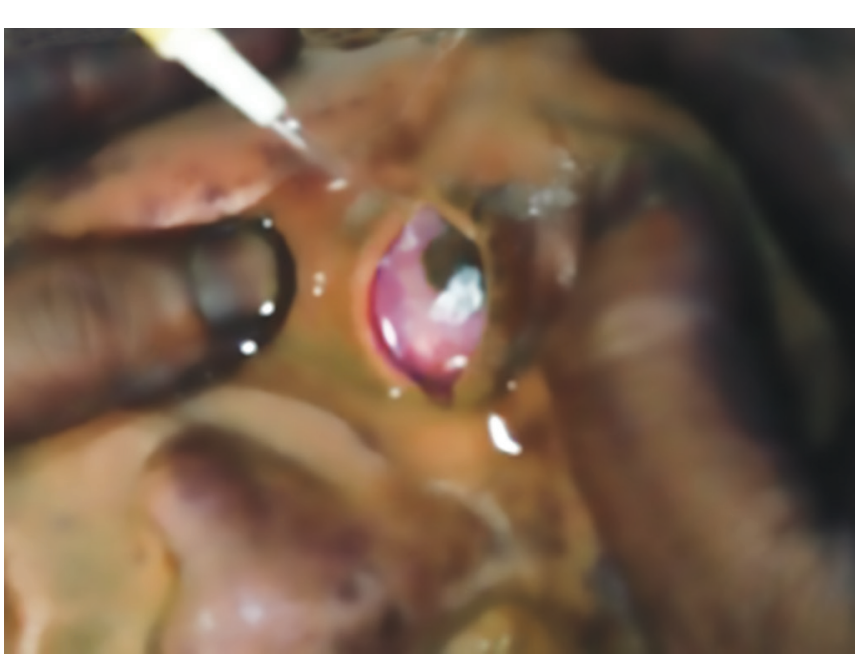

Fig. 2: Saline wash

for patients with color injury. Two doctors and two nurses were posted in the casualty for 24 hours. Holi injured patients were examined by the doctors on slit lamp, ophthalmoscope, and visual acuity/ultrasonography was done. Patients reporting with trauma were admitted.

In cases of color injury, saline wash (Figs 2 and 3) was given thoroughly to remove the foreign particles with prescription of sodium citrate (thimerosal, aqua, sodium citrate) and artificial tears (aqua, glycerol, sodium chloride, and thimerosal) eye drops which are exclusively manufactured the center for Diwali and Holi festival. They were further given prescription containing other medicines as per their requirements.

\section{Open Interview with Patients}

The patients were asked about their demographic details, such as name, age, residence, occupation, and clinical details, such as history of exposure to the harmful chemical, status of vision, duration of exposure, time of first consultation, soundness of mind, and history of referral from other institutions, history of first aid (if any), and outcome of treatment. In phase I, a patient of color injury presented with complaints of burning sensation and blurred vision after exposure to Holi color. In phase II, 25 patients reported to Ophthalmology Casualty in RPC, AIIMS, after 2:00 pm in a disturbed condition after Holi celebration. Patients with color injury had similar complaints as mentioned in phase I. Out of three cases of trauma, two cases were accidentally hit by a balloon and an egg directly in the eye, while another case of trauma was reported where a man had been hit by a cow in an intoxicated state (Fig. 4). There was one case of assault where a man was beaten up in a crowd where Holi was being celebrated on the ground of personal grudge (Table 1).

In phase III, two cases reported for color injury after 2 days of Holi celebration; they were referred from other

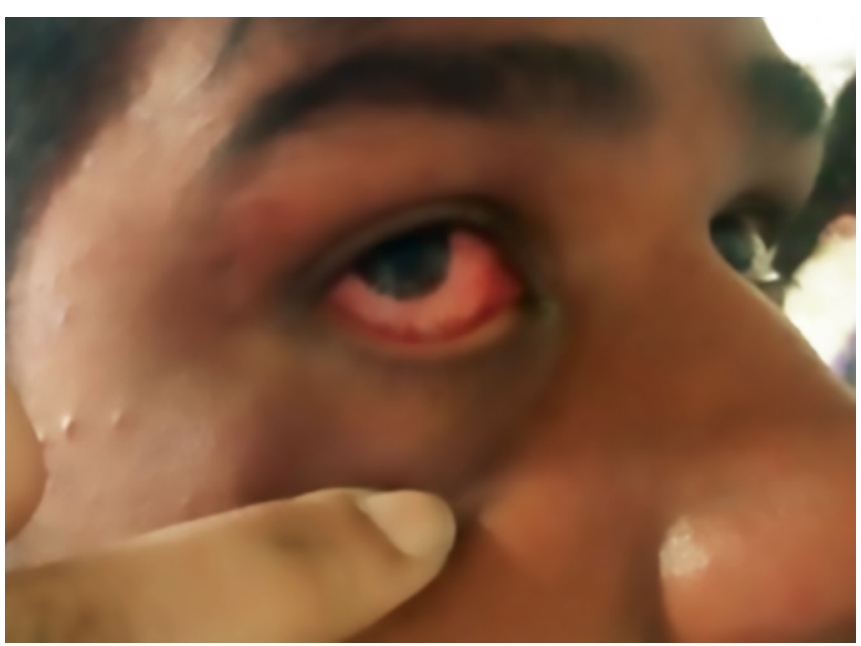

Fig. 3: Color injury

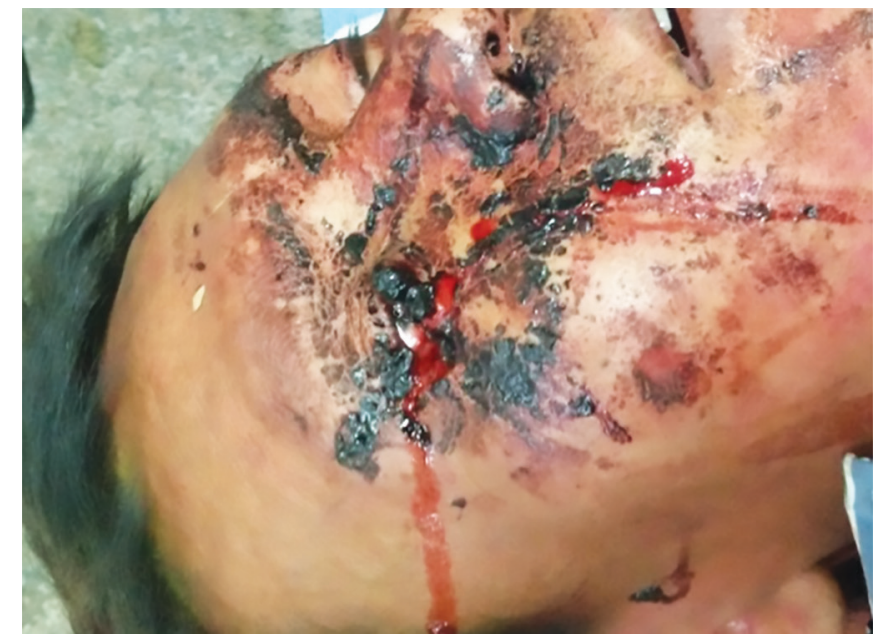

Fig. 4: A case of trauma under substance abuse

hospitals after receiving preliminary care. These patients were interviewed to assess the reason for delay in reporting to AIIMS casualty. On interviewing, it was found that the delay was determined by two factors: Distance and ignorance of patients.

\section{Record Review}

The data were recorded by interviewing the patients coming to the casualty between 8 am and $10 \mathrm{pm}$ and from the records maintained in the record files and computerized data entries.

\section{RESULTS AND OBSERVATION}

A total of 210 patients visited the ophthalmology casualty during the period of study (5 days) out of which 29 (13.81\%) were affected from the events related to Holi. The data of number of patients visiting ophthalmology casualty are depicted in Graph 1. It was observed that there was a sudden increase in cases due to chemical injury during the period of Holi as shown in Graph 2. 
Table 1: Demographic and clinical profile of patients

\begin{tabular}{|c|c|c|}
\hline \multicolumn{3}{|l|}{ Demographic profile } \\
\hline \multirow[t]{2}{*}{ Gender } & Male & 20 \\
\hline & Female & 9 \\
\hline \multirow[t]{2}{*}{ Residence } & Within Delhi & 22 \\
\hline & NCR region & 7 \\
\hline \multirow[t]{3}{*}{ Age group (years) } & $5-20$ & 20 \\
\hline & $20-60$ & 9 \\
\hline & Above 60 & - \\
\hline \multirow[t]{4}{*}{ Occupation } & Student & 15 \\
\hline & Working class & 10 \\
\hline & Housewife & 3 \\
\hline & Retired & 1 \\
\hline \multicolumn{3}{|l|}{ Clinical findings } \\
\hline \multirow[t]{4}{*}{ Cause of injury } & $\begin{array}{l}\text { Color injury from excessive } \\
\text { playing }\end{array}$ & 26 \\
\hline & Assault & 1 \\
\hline & Balloon injury & 1 \\
\hline & Trauma & 1 \\
\hline \multirow[t]{2}{*}{ Status of subject } & Intoxicated & 1 \\
\hline & Sound & 28 \\
\hline \multirow[t]{3}{*}{ Referred cases } & Family physician & 7 \\
\hline & Chemist & 3 \\
\hline & Trauma center & 3 \\
\hline \multirow[t]{2}{*}{ First aid } & None & 18 \\
\hline & Eye drops (in color injury) & 12 \\
\hline \multirow[t]{3}{*}{ Time of reporting } & Within hours & 27 \\
\hline & Next day & 1 \\
\hline & After 2 days & 1 \\
\hline \multirow[t]{2}{*}{ Outcome of treatment } & Improved & 27 \\
\hline & Admitted & 2 \\
\hline \multirow[t]{3}{*}{ Status of vision } & Blurred vision & 23 \\
\hline & Temporary blindness & 6 \\
\hline & Permanent blindness & - \\
\hline \multirow[t]{2}{*}{ First aid } & Premedicated & 12 \\
\hline & Nonmedicated & 17 \\
\hline
\end{tabular}

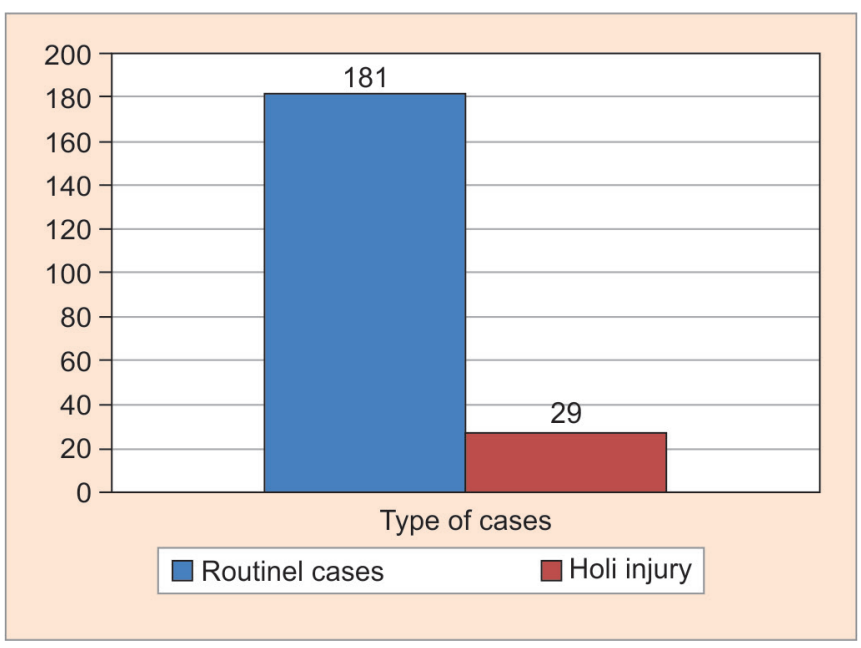

Graph 1: Type of cases reported during Holi

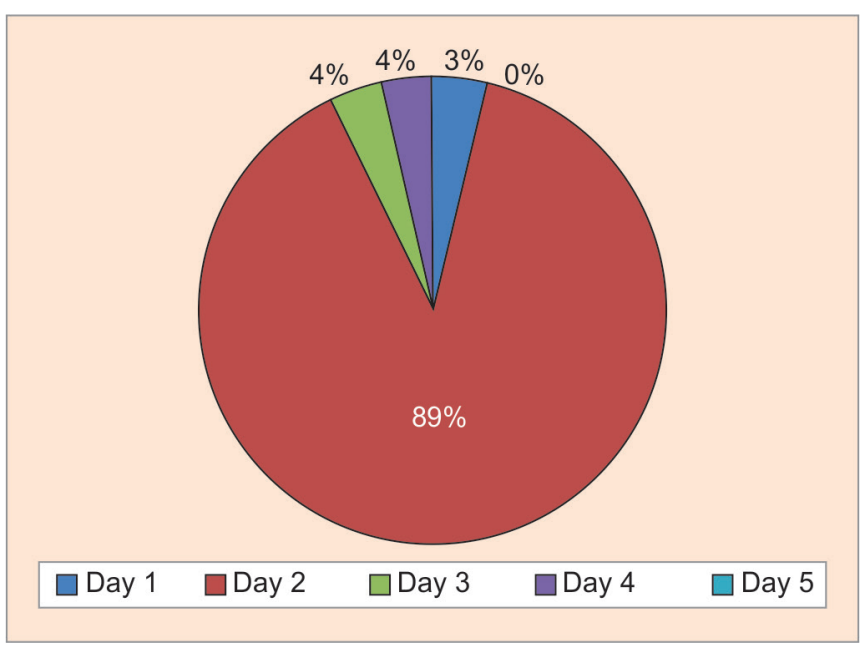

Graph 2: On day 5, out of 55, there were no Holi affected cases

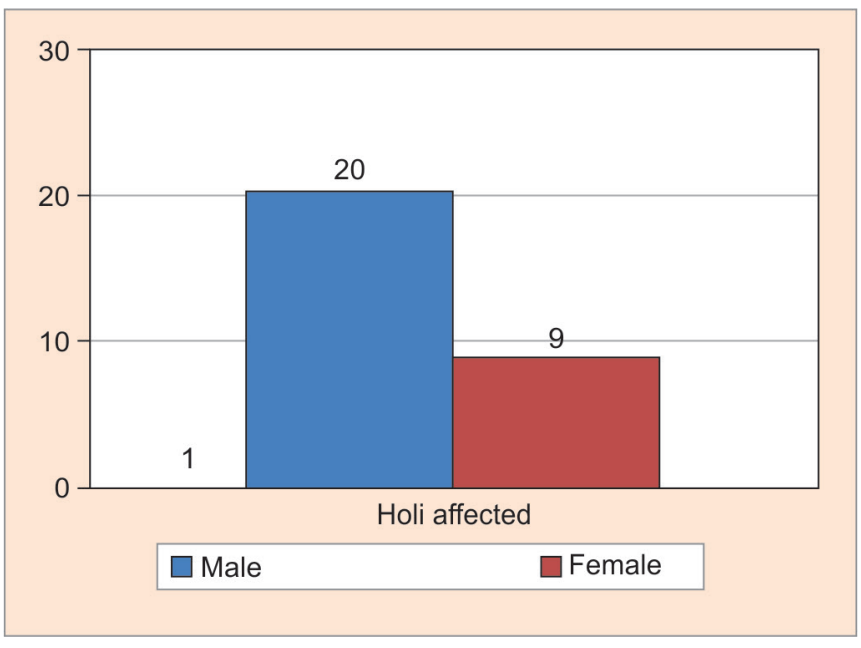

Graph 3: Gender distribution

It shows that maximum cases were reported on day 2 , where out of 45 patients, $26(57.78 \%)$ were cases affected due to Holi followed by day 1 where out of 43, there was 1 $(0.23 \%)$ case of color injury. On days 3 and 4 , out of 44 and 22 patients respectively, one case of color injury in each was reported, constituting 0.23 and $0.45 \%$ respectively. Out of 210 cases reported to ophthalmology casualty during the study period, 129 cases $(68.97 \%)$ were males and 81 cases $(31.03 \%)$ were females. This is depicted in Graph 3.

Graph 4 deals with occupation of patients showing that $51.72 \%$ of cases affected by color injury were students, followed by $34.48 \%$ from service class, $10.34 \%$ cases were housewives, and $3.45 \%$ were retired personnel. The data show that out of 29 Holi affected cases, $86.21 \%$ were affected from color injury, $3.45 \%$ from assault, $6.9 \%$ trauma cases, and $3.45 \%$ from balloon injury as depicted in Graph 5.

By interview of patients, it was found that the average duration from the time of exposure till the time of 


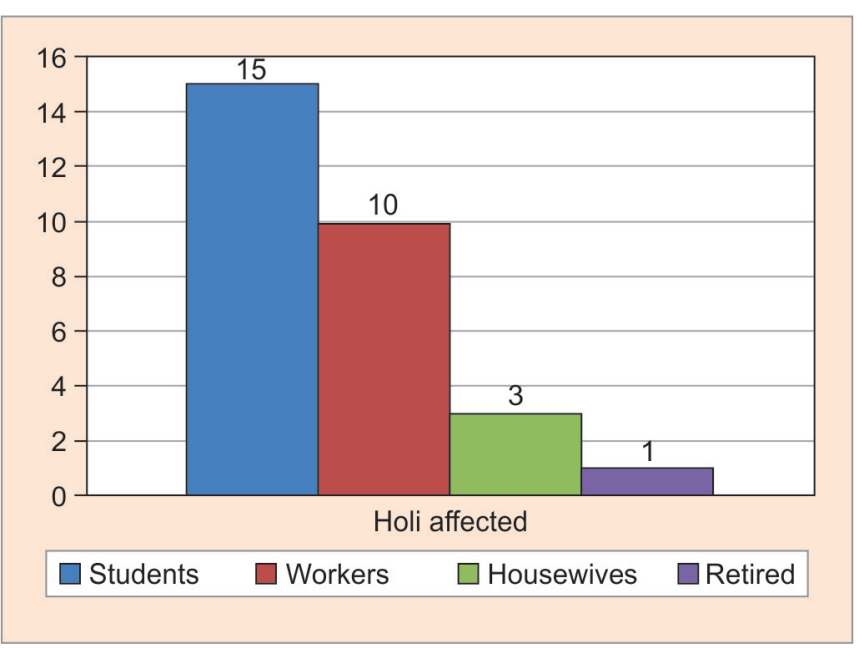

Graph 4: Occupation-based comparison

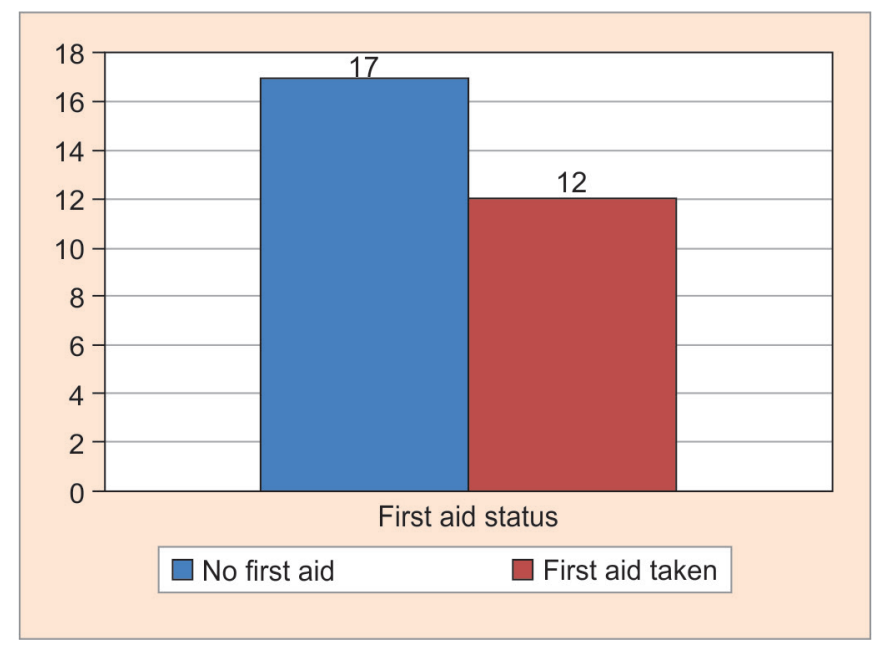

Graph 6: Pretreated and nontreated patients

examination by doctors was found to be 7.9 hours, with minimum time being 1.5 hours and maximum being 48 hours from the time of occurrence of incident.

By analysis of the data, it was found that most incidents of ocular injury during Holi occurred at around $1 \mathrm{pm}$. Earliest exposure was observed at 9 am to as late as $7 \mathrm{pm}$. Average time of reporting to the hospital by the patients was at $4 \mathrm{pm}$ who were examined within 5 minutes. A maximum delay of 60 minutes was observed because of an emergency trauma case which was reported in between.

Thirteen patients were referred from other centers out of which $3(10.34 \%)$ were referred from the associated trauma center of AIIMS, 7 (24.17\%) from family doctors, and $3(10.34 \%)$ others were from nearby chemists as shown in Graph 5.

In all, $41.48 \%$ of patients (12) who reported had already been treated with some unnamed eye drops. Out of 29 patients treated at the ophthalmic casuality, 93\% improved while 3\% were admitted; $94 \%$ patients complained of blurred vision and $6 \%$ suffered from temporary blindness without any serious complication.

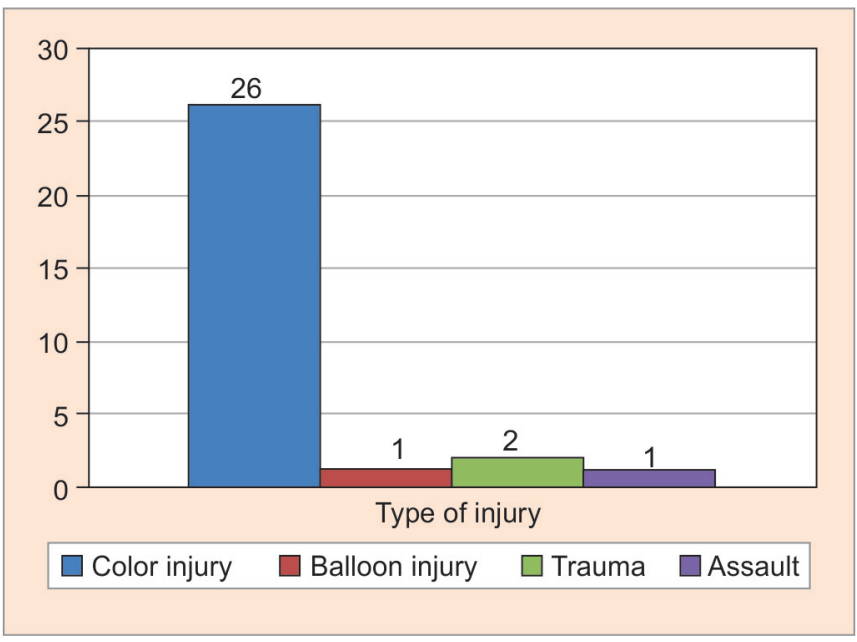

Graph 5: Types of injuries

Over the years, the trend of color-related injury has declined from 41 cases in the year 2016 to 28 in 2017, to 25 in 2018 as seen in Graph 6.

\section{DISCUSSION}

Annually, general outpatient department patient load is $3,63,445$ and in casualty, it is about 56,059 , which suggests that in routine days, number of patients in casualty is approximately 4,671 per month. In the current study period of 5 days, the patient load in the casualty department was 210, which included cases affected due to Holi along with other routine cases like glaucoma, increased IOP, foreign body, orbital cellulitis, and corneal ulcer with sudden rise in cases of chemical injury.

A case study conducted by Susman on water balloon slingshots showed that 17 cases were affected from ocular injury due to water balloon slingshots; in this study, there was one patient who had suffered similarly. Another case study conducted by Ghose et $\mathrm{al}^{3}$ on ocular chemical injuries stated that out of 50 cases of chemical injury, $28 \%$ were due to Holi color and $8 \%$ due to trauma, having $86 \%$ males with a mean age of 25.4 years \pm 12.2 . In the current study, similar results were obtained where majority of color injury cases were seen in young men $(68 \%)$ of same mean age (21.4 years \pm 10$)$.

Another study conducted by Chauhan et $\mathrm{al}^{10}$ on bilateral periorbital necrotizing fasciitis following exposure to Holi color presented a case of a 57-year-old patient suffering from complications after being exposed to Holi color; however, in this study, no complications were observed.

At other hospitals (Delhi, Uttar Pradesh, Haryana), the number of cases reporting to emergency departments on the day of Holi was above 770, which included all the cases of road accidents, murders, and assault, although 2018 has marked lowest road deaths on Holi 


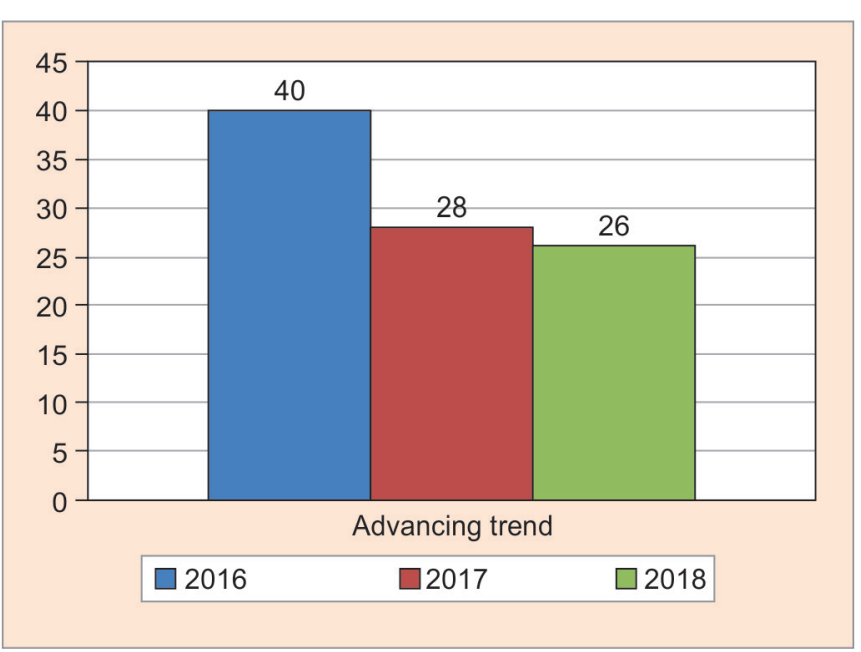

Graph 7: Comparison with data of previous years

in Delhi over 7 years. On the day of Holi, over 9,300 people in Delhi were booked for traffic violations out of which 1,300 cases were caught by Delhi police for drunken driving.

\section{CONCLUSION AND RECOMMENDATIONS}

It is concluded that

- Routine patient flow during Holi and other days in casualty has remained static with a sudden increase in cases of chemical injury.

- There has been a decline in the number of color injury cases over the past 3 years (Graph 7).

- A significant decrease in cases of trauma and assault cases, implying better enforcement of laws.

- There has been minimal extent of damage to eyes because of immediate reporting time, which implies an increase in the number of more informed and learned public.

- Holi is a joyous festival and should be played with utmost precautions by using herbal colors, protective glasses, oiling of hair and skin, and if a harmful exposure occurs, nearby hospital should be sought for treatment without delay.

\section{REFERENCES}

1. White Planet Technologies. Indian festivals. India: White Planet Technologies; 2017. [cited 2018 Mar 15]. Available from: http://www.indiacelebrating.com/festivals-of-india/.

2. Royal College of Ophthalmologists. What is ophthalmology? London: Royal College of Ophthalmologists; 2017. [cited 2018 Mar 15]. Available from: https://www.rcophth.ac.uk/about/ what-is-ophthalmology/.

3. Ghose, S.; Titiyal, JS.; Sharma, N.; Velpandian, T.; Rajpal. Ocular chemical injuries: chemical characterizations and clinical profile correlations. Cornea session III: AIOC 2010 Proceedings, New Delhi. 2010. [cited 2018 Apr 5]. pp. 258-260.

4. Staver, J. Common injuries caused by water balloons. Chicago (IL): Staver Law Group; [cited 2018 Apr 5]. Available from: https://www.chicagolawyer.com/common-injuriescaused-water-balloons/.

5. The Hindu. Substance abuse cases rise during Holi. New Delhi: The Hindu; 2006. [cited 2018 Apr 5]. Available from: http://www.thehindu.com/todays-paper/tp-national/ tp-newdelhi/substance-abuse-cases-on-the-rise-aroundholi/article3164807.ece.

6. National Programme for Control of Blindness, Government of India. Welcome to national programme for control of blindness. New Delhi: NPCB; [cited 2018 Apr 2]. Available from: http://www.npcb.nic.in/.

7. Bossmann K, Bach S, Höflich C, Valtanen K, Heinze R, Neumann A, Straff W, Süring K. Holi colours contain PM10 and can induce pro-inflammatory responses. J Occup Med Toxicol 2016 Sep;11(1):42.

8. Susman, ED. Case study on ocular effects of water balloon slingshots. 1995. [cited 2018 Apr 5].

9. Velpandian T, Saha K, Ravi AK, Kumari SS, Biswas NR, Ghose S. Ocular hazards of the colors used during the festival-of-colors (Holi) in India-malachite green toxicity. J Hazard Mater 2007 Jan;139(2):204-208.

10. Chauhan D, Arora R, Das S, Shroff D, Narula R. Bilateral periorbital necrotizing fasciitis following exposure to Holi colors: a case report. Indian J Ophthalmol 2007 Sep-Oct;55(5):373-374. 